\title{
Profound metabolic acidosis from pyroglutamic acidemia: an underappreciated cause of high anion gap metabolic acidosis
}

\author{
Thomas J. Green, MD, MSc; ${ }^{*}$ Jan Jaap Bijlsma, MD; ; David D. Sweet, MD ${ }^{*}+\neq$
}

\section{ABSTRACT}

The workup of the emergency patient with a raised anion gap metabolic acidosis includes assessment of the components of "MUDPILES" (methanol; uremia; diabetic ketoacidosis; paraldehyde; isoniazid, iron or inborn errors of metabolism; lactic acid; ethylene glycol; salicylates). This approach is usually sufficient for the majority of cases in the emergency department; however, there are many other etiologies not addressed in this mnemonic. Organic acids including 5-oxoproline (pyroglutamic acid) are rare but important causes of anion gap metabolic acidosis. We present the case of a patient with profound metabolic acidosis with raised anion gap, due to pyroglutamic acid in the setting of malnutrition and chronic ingestion of acetaminophen.

Keywords: pyroglutamic acid, 5-oxoproline, metabolic acidosis, acetaminophen, malnutrition, case report

\section{RÉSUMÉ}

L'investigation d'un patient à l'urgence présentant une acidose métabolique à trou anionique augmenté comprend l'évaluation des composantes de "MUDPILES " (mnémonique anglais pour méthanol; urémie; acidocétose diabétique; paraldéhyde; fer ou maladie enzymatique; acide lactique; éthylène glycol; salicylate). Cette approche suffit généralement pour la majorité des cas à l'urgence, mais ce mnémonique ne tient pas compte de nombreuses autres étiologies. Les acides organiques, dont le 5-oxoproline (acide L-pyroglutamique) sont des causes rares mais importantes d'acidose métabolique à trou anionique. Nous présentons ici le cas d'une patiente présentant une acidose métabolique profonde à trou anionique augmenté, en raison de présence d'acide L-pyroglutamique dans un contexte de malnutrition et d'ingestion chronique d'acétaminophène.

\section{CASE REPORT}

A 43-year-old woman was transported via ambulance to the emergency department (ED) after being found screaming incoherently in the hallway of her apartment building. On arrival she was restless and remained incoherent. She was accompanied by friends who confirmed that she had been in her usual state the previous day; however, she had recently received treatment at an addiction treatment centre for prescription narcotic dependence. She had lost $50 \mathrm{lb}$ during the prior 6 months because of an eating disorder, and was likely malnourished. During her stay at the addiction treatment centre and in the days since her discharge, she had been ingesting large amounts of acetaminophen and a number of empty acetaminophen bottles were found in her apartment.

Her medical history included recurrent nephrolithiasis and chronic lower back pain as well as a remote history of cocaine and alcohol abuse. Her other medications included quetiapine, trazodone, methadone, a stimulant laxative, docusate, gabapentin, furosemide and citalopram.

Physical examination revealed a very thin woman with a body mass index of 18 . Her temperature was $36.4^{\circ} \mathrm{C}$, heart rate was 95 beats/min, blood pressure was 109/70 mm Hg, respiratory rate was 30 breaths/min and Glasgow Coma Scale score was 14 (eyes 4, verbal 4, motor 6). Her pupils were equal at $4 \mathrm{~mm}$ and reactive to light. Respiratory examination showed tachypnea, but was otherwise unremarkable. Her cardiovascular and abdominal examinations were normal. There were no focal neurologic findings and

From the Departments of *Emergency Medicine and TMedicine, University of British Columbia, Vancouver, BC, and the $¥$ Division of Critical Care Medicine, Vancouver General Hospital, Vancouver, BC

Submitted Apr. 26, 2009; Revised Oct. 3, 2009; Accepted Nov. 29, 2009

This article has been peer reviewed.

CJEM 2010;12(5):449-52 
no signs of trauma or infection. There were no physical signs of a specific toxidrome.

Laboratory values were as follows: sodium $141 \mathrm{mmol} / \mathrm{L}$, potassium $3.1 \mathrm{mmol} / \mathrm{L}$, chlorine $102 \mathrm{mmol} / \mathrm{L}$, carbon dioxide $5 \mathrm{mmol} / \mathrm{L}$, glucose $6.8 \mathrm{mmol} / \mathrm{L}$ and urea $10.6 \mathrm{mmol} / \mathrm{L}$, equating to a profound anion gap of $34 \mathrm{mmol} / \mathrm{L}$. Serum creatinine was elevated at $218 \mu \mathrm{mol} / \mathrm{L}$. Arterial blood gas testing on high flow oxygen showed a $\mathrm{pH}$ level of 7.11, a partial pressure of carbon dioxide of $14 \mathrm{~mm} \mathrm{Hg}$, a partial pressure of oxygen of $350 \mathrm{~mm} \mathrm{Hg}$ and a bicarbonate level of $4 \mathrm{mmol} / \mathrm{L}$, in keeping with a substantial metabolic acidosis. Thyroid-stimulating hormone level was normal, white blood cell count was $19.9 \times 10^{9} / \mathrm{L}$ and hemoglobin level was $129 \mathrm{~g} / \mathrm{L}$. Transaminase levels were slightly elevated (alanine transaminase: $3.56 \mu \mathrm{kat} / \mathrm{L}$ [213 U/L]; aspartate transaminase: $1.02 \mu \mathrm{kat} / \mathrm{L}$ [61 U/L]) and lipase level was $7.8 \mu \mathrm{kat} / \mathrm{L}$ (469 U/L). Her serum acetaminophen level was $399 \mu \mathrm{mol} / \mathrm{L}$. A computed tomographic scan of her head was normal and electrocardiography revealed a narrow complex sinus rhythm. Urinalysis and urine toxicology testing showed no evidence of abnormality or ingestion.

Workup for anion gap metabolic acidosis revealed a normal lactate of $1.7 \mathrm{mmol} / \mathrm{L}$ and measured serum osmolality of $305 \mathrm{mmol} / \mathrm{kg}$ with an osmolar gap of only $5.6 \mathrm{mmol} / \mathrm{kg}$. Results of testing for serum ketones, acetylsalicylic acid, ethylene glycol, methanol and ethanol were negative. There was no history of ingestion of iron, paraldehyde or isoniazid.

The patient was referred to the intensive care unit for management of profound metabolic acidosis. Her treatment included intravenous $N$-acetylcysteine, a bolus of sodium bicarbarbonate followed by an infusion, as well as empiric treatment with fomepizole, thiamine, folate and ceftriaxone. She received continuous venovenous hemodialysis overnight for the unexplained metabolic acidosis. By the following morning her acidosis had resolved, as had her state of confusion. The dialysis and $\mathrm{N}$-acetylcysteine were stopped. The patient remained in hospital for 4 days and recovered completely.

A urine sample collected during her initial emergency presentation was tested for organic acid levels. The sample had a profoundly elevated level of 5-oxoproline of 17292 $\mu \mathrm{mol} / \mathrm{L}$ creatinine, which was more than 200 times the reported upper limit of normal $(79 \mu \mathrm{mol} / \mathrm{L}$ creatinine $)$.

\section{DISCUSSION}

Pyroglutamic acid is an intermediate in the $\gamma$-glutamyl cycle, which is involved in transport of amino acids across the cell membrane and in the synthesis of glutathione. Pyroglutamic aciduria was initially described in pediatric literature resulting from autosomal recessive deficiencies of glutathione synthetase ${ }^{1}$ or 5 -oxoprolinase. ${ }^{2,3}$ A syndrome of acquired pyroglutamic metabolic acidosis was first described in an adult in $1989^{4}$ and since then a number of individual case series have been published..$^{5-7}$ The disorder has been associated with ingestion of a number of medications, including vigabatrin, ${ }^{8}$ netilmicin ${ }^{9}$ and flucloxacillin. ${ }^{7,9-13}$ In the majority of patients there is also ingestion or co-ingestion of acetaminophen. ${ }^{7,11-18}$ Although some patients ingested supratherapeutic doses of acetaminophen, the majority received only therapeutic doses for pain or fever during lengthy hospital admissions.

A common thread among case reports of pyroglutamic acidosis is its association with critical illness and significant comorbidities, including sepsis and renal failure. ${ }^{14}$ The typical presentation occurs in a patient who develops an acutely altered level of consciousness and the clinical manifestations of metabolic acidosis during a prolonged hospital stay. There are, however, cases of patients presenting from home, ${ }^{5-7,14,15,17,18}$ and our case supports evidence that patients can present without significant comorbidity other than malnutrition. It is important to note that our patient's pyroglutamic metabolic acidosis was in the context of chronic acetaminophen use in the face of malnutrition and was not associated with the metabolic acidosis seen in acetaminophen overdose that is usually accompanied by hepatic and renal failure.

Metabolic acidosis is associated with acetaminophen overdose and was first reported in $1970 .{ }^{19}$ In that setting, the acidosis is more commonly driven by lactic acidemia in the context of hepatic failure. In fact, metabolic acidosis directly resulting from acetaminophen overdose is thought of as a late development, a poor prognostic indicator and one of the King's College Criteria for hepatic transplant. ${ }^{20}$ Acetaminophen-related pyroglutamic acidosis arises from an independent mechanism and does not carry the same prognostic implications. Interestingly, there have been cases of "unexplained" metabolic acidosis occurring early in acetaminophen overdose, unrelated to lactic acidosis, renal or hepatic failure. ${ }^{19,21}$ It is plausible that a number of these cases resulted from unrecognized pyroglutamic acidosis.

The mechanism by which medication and comorbidities induce pyroglutamic acidosis is not fully understood. Based on animal studies ${ }^{22}$ and knowledge of the $\gamma$-glutamyl cycle a plausible theory has been proposed. ${ }^{6}$ As seen in Figure 1, a deficiency of glutathione removes a negative 
feedback point, resulting in excess $\gamma$-glutamylcysteine, some of which is shunted by $\gamma$-glutamylcyclotransferase to reform pyroglutamic acid. The excess pyroglutamic acid overwhelms the rate limiting 5-oxoprolinase enzyme and spills over, contributing to pyroglutamic metabolic acidosis. This mechanism may explain how glutathione deficiency, as seen in sepsis, critical illness, nutritional deficiency and chronic acetaminophen ingestion, may lead to pyroglutamic acidemia.

Diagnosis of pyroglutamic acidosis is based on clinical suspicion, as the slow turnaround time of confirmatory urine testing for organic acids limits its diagnostic utility to emergency physicians. Treatment is theoretical and is guided by principles of supportive management of severe acidosis. Hemodialysis effectively clears pyroglutamic acid and may be indicated. Based on the role of glutathione deficiency in this disorder, the use of $\mathrm{N}$-acetylcysteine, a glutathione donor, is a plausible treatment. ${ }^{6}$ There is no evidence to support any dosing regimen, but our patient was treated with the standard intravenous protocol for acetaminophen overdose.

It is important that emergency physicians recognize that there are causes of metabolic acidosis that are not addressed in the common "MUDPILES" (methanol; uremia; diabetic ketoacidosis; paraldehyde; isoniazid, iron or inborn errors of metabolism; lactic acid; ethylene glycol; salicylates) mnemonic. Beside organic acids such as pyroglutamic acid, other causes such as D-lactate, which results from short bowel syndrome after bowel resection ${ }^{23}$ and is not measured by conventional laboratory tests, should be considered. In cases of suspected pyroglutamic acid, the emergency physician can be instrumental in facilitating diagnosis by testing urine for organic acids, as well as beginning therapeutic management with bicarbonate and $N$-acetylcysteine and mobilizing resources such as dialysis to better care for patients.

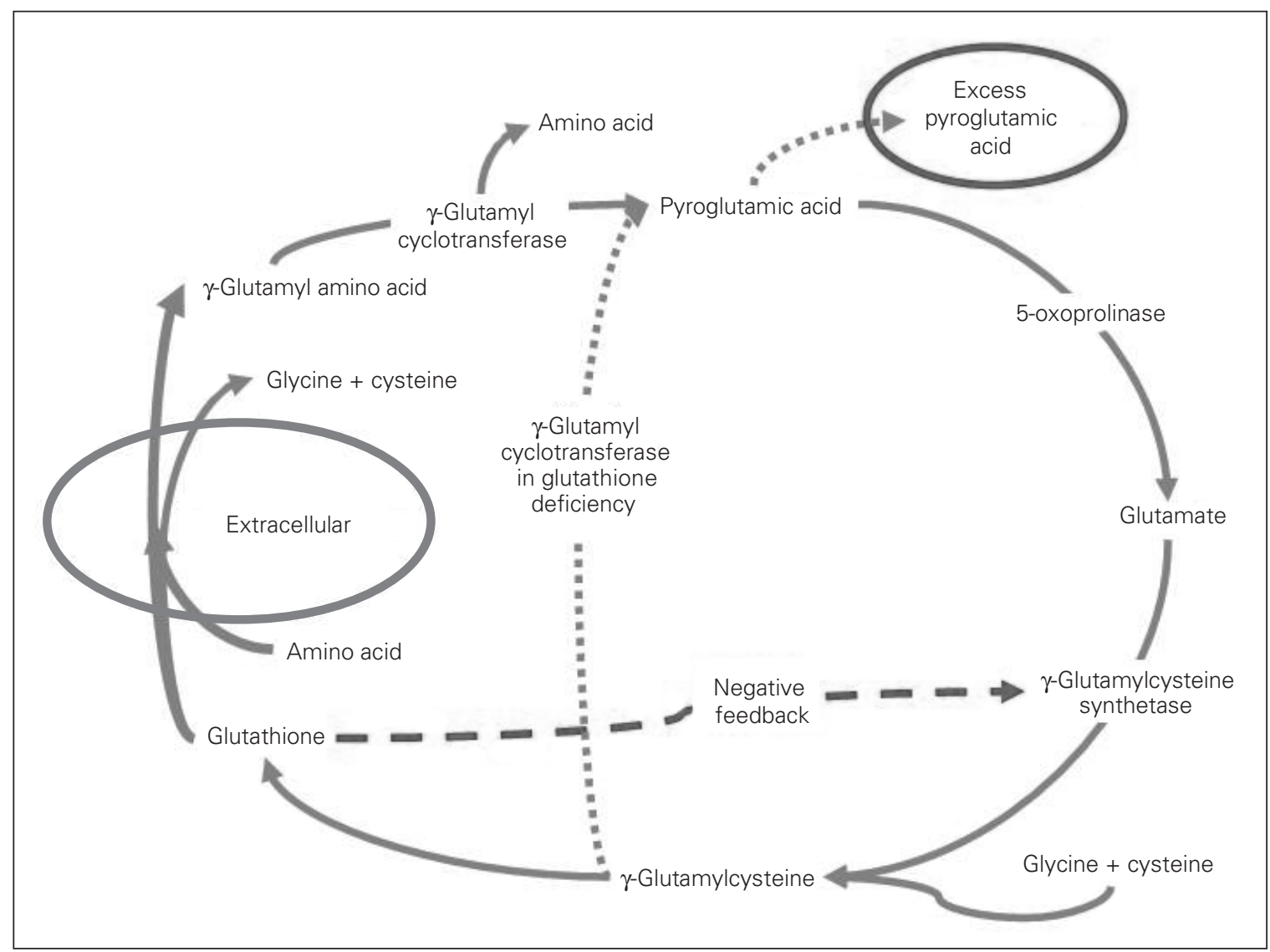

Fig. 1. In the extracellular space, glutathione combines with an amino acid, releasing glycine and cysteine, and is then transported into the cell. Inside the cell, $\gamma$-glutamyl cyclotransferase removes the transported amino acid, producing pyroglutamic acid (5-oxoproline) and then glutathione is regenerated over a number of steps. Deficiency of glutathione removes a negative feedback point, resulting in excess $\gamma$-glutamylcysteine. In this circumstance, $\gamma$-glutamyl cyclotransferase, the enzyme involved earlier in the cycle to remove the transported amino acid, can metabolize $\gamma$-glutamylcysteine to reform pyroglutamic acid. Excess pyroglutamic acid overwhelms the rate, limiting 5-oxoprolinase and results in pyroglutamic metabolic acidosis. 


\section{CONCLUSION}

Pyroglutamic acidemia is an important, although uncommon, cause of profound anion gap metabolic acidosis. The literature is limited to case reports, and our knowledge of contributory comorbidities, medications and the contribution of underlying genetics remains incomplete. It is possible that this disorder occurs more frequently in less severe cases and is therefore underappreciated. Although the majority of described cases occur in admitted patients with significant comorbidities, our case describes pyroglutamic acidosis in a malnourished patient presenting to the ED from home. In the ED, most instances of anion gap metabolic acidoses can be investigated and managed with the traditional MUDPILES approach; however, it is important to recognize the existence of less common causes of anion gap acidosis. Finally, it is important to understand that, in the context of acetaminophen ingestion, metabolic acidosis from pyroglutamic acid arises from a mechanism independent of hepatic failure, and is not a poor prognostic indicator.

Competing interests: None declared.

\section{REFERENCES}

1. Wellner VP, Sekura R, Meister A, et al. Glutathione synthetase deficiency, an inborn error of metabolism involving the gamma-glutamyl cycle in patients with 5-oxoprolinuria (pyroglutamic aciduria). Proc Natl Acad Sci U S A 1974;71:2505-9.

2. Larsson A, Mattsson B, Wauters EA, et al. 5-oxoprolinuria due to hereditary 5-oxoprolinase deficiency in two brothers a new inborn error of the gamma-glutamyl cycle. Acta Paediatr Scand 1981;70:301-8.

3. Roesel RA, Hommes FA, Samper L. Pyroglutamic aciduria (5-oxoprolinuria) without glutathione synthetase deficiency and with decreased pyroglutamate hydrolase activity. 7 Inherit Metab Dis 1981;4:89-90.

4. Creer MH, Lau BW, Jones JD, et al. Pyroglutamic acidemia in an adult patient. Clin Chem 1989;35:684-6.

5. Fenves AZ, Kirkpatrick HM III, Patel VV, et al. Increased anion gap metabolic acidosis as a result of 5-oxoproline (pyroglutamic acid): a role for acetaminophen. Clin $7 \mathrm{Am}$ Soc Nephrol 2006;1:441-7.

6. Pitt JJ, Hauser S. Transient 5-oxoprolinuria and high anion gap metabolic acidosis: clinical and biochemical findings in eleven subjects. Clin Chem 1998;44:1497-503.

7. Dempsey GA, Lyall HJ, Corke CF, et al. Pyroglutamic acidemia: a cause of high anion gap metabolic acidosis. Crit Care Med 2000;28:1803-7.

8. Bonham JR, Rattenbury JM, Meeks A, et al. Pyroglutamicaciduria from vigabatrin. Lancet 1989;1:1452-3.

9. Croal BL, Glen AC, Kelly CJ, et al. Transient 5-oxoprolinuria (pyroglutamic aciduria) with systemic acidosis in an adult receiving antibiotic therapy. Clin Chem 1998;44:336-40.

10. Peter JV, Rogers N, Murty S, et al. An unusual cause of severe metabolic acidosis. Med J Aust 2006;185:223-5.

11. Brooker G, Jeffery J, Nataraj T, et al. High anion gap metabolic acidosis secondary to pyroglutamic aciduria (5-oxoprolinuria): association with prescription drugs and malnutrition. Ann Clin Biochem 2007;44:406-9.

12. Rolleman EJ, Hoorn EJ, Didden P, et al. Guilty as charged: unmeasured urinary anions in a case of pyroglutamic acidosis. Neth 7 Med 2008;66:351-3.

13. Kortmann W, van Agtmael MA, van Diessen J, et al. 5-Oxoproline as a cause of high anion gap metabolic acidosis: an uncommon cause with common risk factors. Neth J Med 2008; 66:354-7.

14. Foot CL, Fraser JF, Mullany DV. Pyroglutamic acidosis in a renal transplant patient. Nephrol Dial Transplant 2005;20: 2836-8.

15. Hodgman MJ, Horn JF, Stork CM, et al. Profound metabolic acidosis and oxoprolinuria in an adult. 7 Med Toxicol 2007;3:119-24.

16. Humphreys BD, Forman JP, Zandi-Nejad K, et al. Acetaminophen-induced anion gap metabolic acidosis and 5-oxoprolinuria (pyroglutamic aciduria) acquired in hospital. Am J Kidney Dis 2005;46:143-6.

17. Pitt JJ, Brown GK, Clift V, et al. Atypical pyroglutamic aciduria: possible role of paracetamol. J Inherit Metab Dis 1990; 13:755-6.

18. Tailor P, Raman T, Garganta CL, et al. Recurrent high anion gap metabolic acidosis secondary to 5-oxoproline (pyroglutamic acid). Am J Kidney Dis 2005;46:e4-10.

19. Proudfoot AT, Wright N, Proudfoot AT, et al. Acute paracetamol poisoning. BMJ 1970;3:557-8.

20. O'Grady JG, Alexander GJ, Hayllar KM, et al. Early indicators of prognosis in fulminant hepatic failure. Gastroenterology 1989;97:439-45.

21. Zezulka A, Wright N, Zezulka A, et al. Severe metabolic acidosis early in paracetamol poisoning. Br Med J (Clin Res Ed) 1982;285:851-2.

22. Ghauri FY, McLean AE, Beales D, et al. Induction of 5-oxoprolinuria in the rat following chronic feeding with $\mathrm{N}$-acetyl 4-aminophenol (paracetamol). Biochem Pharmacol 1993;46: 953-7.

23. Petersen C. D-lactic acidosis. Nutr Clin Pract 2005;20:634-45.

Correspondence to: Dr. Thomas Green, Department of Emergency Medicine, Vancouver General Hospital, 910 West 10th Ave., ground floor, JPPN, Vancouver BC V5Z 4E3; tomgreen@interchange.ubc.ca 